\title{
El modelo cinético-corpuscular y las prácticas científicas: una propuesta basada en la dilatación térmica
}

\author{
María Roser Nebot Castelló y Conxita Márquez Bargalló \\ Grupo LIEC, Departamento de Didáctica de las Ciencias. UAB. \\ Cerdanyola del Vallès, España
}

[Recibido el 27 de octubre de 2018, aceptado el 24 de marzo de 2019]

En el artículo se discuten los resultados del análisis de un examen de cuatro grupos de alumnos de 11-12 años, dos con una profesora novel y dos con una experimentada. Se analiza la utilización de ideas clave del modelo cinético-corpuscular en dibujos, descripciones y explicaciones de fenómenos de dilatación térmica. En los dibujos se analiza la representación de la separación y movimiento de las partículas. Se detectan más dificultades en el alumnado de la profesora novel, y más en hacerlos que en explicar lo observado en dibujos ya hechos. En las descripciones, se diferencia entre el alumnado que solo indica lo observado y el alumnado que relaciona los cambios de temperatura con los de volumen. En las explicaciones, se han registrado las ideas clave del modelo utilizadas y se ha considerado adecuado su uso si la causalidad de las ideas es correcta y su expresión coherente. Se han detectado distintos niveles de desempeño, siendo mejores los del alumnado de la profesora experimentada.

Palabras clave: modelización; modelo cinético-corpuscular; prácticas científicas; explicaciones; prácticas de laboratorio.

\section{The kinetic-corpuscular model and the scientific practices: a proposal based on thermal expansion}

This article discusses the results of the analysis of an exam of four groups of 11-12 year-old students, two with an experienced teacher and two with a novice teacher. The use of key ideas of the kinetic-corpuscular model in diagrams was analysed, as well as descriptions and explanations of thermal expansion phenomena. In the drawings, the representation of separation and movement of particles was recorded. There were more difficulties in the students whose teacher was novice, and more in doing them than in explaining what was observed in printed drawings. In the descriptions, there were students that only indicated what they saw, but others included the relationship of temperature and volume changes. In explanations, the use of key ideas of the model was recorded, and they were considered appropriate if the causality of the ideas was correct and its expression was consistent. Different levels of performance were detected, being better those of the students of the experienced teacher.

Keywords: modelling; kinetic-corpuscular model; scientific practices; explanations; laboratory work.

Para citar el artículo. Nebot, MR y Márquez,C. (2019). El modelo cinético-corpuscular y las prácticas científicas: una propuesta basada en la dilatación térmica. Ápice. Revista de Educación Científica, 3(2), 21-35. DOI: https://doi.org/10.17979/arec.2019.3.2.4625

Contacto: mrnebot@gmail.com, Conxita.marquez@uab.cat 


\section{Introducción}

Un problema con el que suele encontrarse el profesorado es la poca capacidad del alumnado para interpretar el comportamiento de los fenómenos naturales y para explicar científicamente aquello que observa o conoce. Las dificultades que muestra el alumnado cuando tiene que expresar los fenómenos que estudia provienen muchas veces del enfoque de las actividades que se proponen. El conocimiento adquirido suele ser superficial, porque normalmente el estudio de los fenómenos se limita a su descripción en términos de propiedades, como cambios de volumen, cambios de temperatura o cambios de estado.

Nuestro estudio se originó porque observamos que el alumnado de 1ㅇ de la ESO mostraba dificultades para interpretar y explicar procesos clave del ciclo del agua, de la dinámica de los ecosistemas o de fisiología, relacionados con el modelo cinético-corpuscular.

En el marco de las Ciencias Experimentales, cuando se trabajan fenómenos del campo de la Biología o la Geología, no suelen realizarse explicaciones basadas en el modelo cinético-corpuscular de la materia. Por ejemplo, se dice que, en la fusión, el agua pasa del estado sólido al líquido, y no se explica cómo se produce el proceso.

Pensamos que para ir más allá de la descripción de lo directamente perceptible es necesario trabajar el modelo cinético-corpuscular, que normalmente no se introduce hasta 20 de la ESO, porque permite explicar y predecir una variedad muy amplia de fenómenos físicos (Benarroch, 2000). Por ello, iniciamos a los alumnos de 10 de la ESO en la visión a nivel de partículas y en la realización de explicaciones científicas. Después de unas actividades previas sobre la naturaleza discreta de la materia y las características de cada estado, trabajamos con procesos de dilatación térmica realizados en prácticas de laboratorio, que permiten observar e interpretar cambios en el comportamiento de los materiales sin cambiar de estado.

En el curso 2016/17, después de haber implementado las actividades durante unos años, se introdujeron cambios en los materiales y se elaboró una lista de ideas clave del modelo, que se usaron para realizar explicaciones a nivel de partículas. Se tuvo en cuenta que, además de trabajar con el modelo cinético-corpuscular, debían integrarse las habilidades lingüísticas asociadas (Lemke, 1997). Para interpretar y explicar los fenómenos no sólo es necesario tener un marco de referencia, un modelo científico, sino que también debe aprenderse a comunicar, a expresar aquello que se aprende. Para ello se dieron pautas lingüísticas para realizar descripciones y explicaciones científicas utilizando las ideas del modelo cinético-corpuscular, que permitieron la interpretación de los fenómenos observados en el laboratorio y de otros fenómenos del mundo natural y de la vida cotidiana.

A partir de los resultados obtenidos en el estudio piloto (Nebot y Márquez, 2017) y su discusión, se hicieron cambios en las actividades y en el examen del curso 2017/18.

\section{Objetivos}

En este artículo se discuten los resultados del análisis de un examen de alumnado de 10 de la ESO del curso 2017/18, sobre el uso del modelo cinético corpuscular en relación a fenómenos de dilatación térmica. Los objetivos son:

1. Analizar las ideas clave que aparecen en dibujos, descripciones y explicaciones que hacen los alumnos al responder preguntas sobre fenómenos de dilatación térmica estudiados en clase.

2. Analizar la aplicación de las ideas clave en explicaciones de fenómenos cotidianos de dilatación térmica no estudiados en clase. 


\section{Marco teórico}

El estudio se fundamenta en los campos teóricos sobre prácticas científicas, concretamente en la modelización y la elaboración de dibujos, descripciones y explicaciones científicas.

\section{Prácticas científicas}

En los NGSS (2013), estándares comunes para la enseñanza de las ciencias en Estados Unidos, se propone trabajar un número limitado de ideas clave integradas entre ellas y con las prácticas científicas, que incluyen hacer preguntas, desarrollar y utilizar modelos, analizar e interpretar datos, elaborar explicaciones y planificar e implementar investigaciones. Osborne (2014) indica que las prácticas científicas involucran a los estudiantes en actividades que permiten construir, profundizar y aplicar su conocimiento de las ideas clave para la construcción de las ideas científicas. De esta manera, el alumnado procede de la misma manera que lo hacen los científicos y aprende cómo se trabaja cuando se estudia un problema científico.

Esta manera de mirar entronca claramente con el modelo cognitivo de ciencia escolar (Izquierdo, Sanmartí y Espinet, 1999; Adúriz-Bravo e Izquierdo-Aymerich, 2009), que considera el modelo teórico como la unidad fundamental de la ciencia, tanto de los científicos como de la ciencia escolar. Destaca que el alumno no es un científico, sino que es un científico escolar, y debemos tener muy en cuenta este aspecto al diseñar las actividades de ciencia escolar. Es por ello que hablaremos de actividad científica escolar (ACE), de la misma manera que hablamos de actividad científica cuando nos referimos a las prácticas de los científicos, y de modelos científicos escolares (MCE) para indicar aquellos que elaboramos o aplicamos para el aprendizaje de nuestros alumnos.

Los MCE no son iguales que los modelos científicos, pero son análogos a ellos: tienen un poder explicativo similar, pero están adaptados a las necesidades de los alumnos y deben evolucionar a medida que avanza el aprendizaje (Garrido, 2016).

La introducción del modelo cinético-corpuscular de la materia suele realizarse con la descripción de la separación y movimiento de las partículas (sin distinguir entre átomos y moléculas) en sólidos, líquidos y gases, pero sin enmarcarlo en una determinada substancia y en los cambios de estado (Johnson y Papageorgiou, 2010). Los autores proponen trabajar los cambios que experimenta una determinada sustancia y relacionarlos con la distribución de energía entre diferentes substancias. La dilatación térmica puede ser una buena iniciación, porque en el laboratorio se observan cambios de volumen de una determinada substancia sin cambiar de estado. Así, los estados se presentan dinámicos y se favorece el estudio de los cambios de estado vistos como un contínuum, como un proceso, no como cambios bruscos.

Cuando observamos fenómenos para poder explicar qué ha pasado y compartirlo con otros, basándonos en un modelo que hemos creado, utilizamos representaciones visuales. Centrándonos en el modelo cinético-corpuscular, las representaciones son de tres tipos: macro, submicro y simbólicas (Gilbert, 2010). Las representaciones macro son descriptivas, porque se basan en propiedades empíricas de las substancias, que se pueden medir con instrumentos a nuestro alcance (Gilbert, Boulter y Elmer, 2000; citados por Gilbert 2010). Las representaciones submicro se refieren a entidades demasiado pequeñas para ser vistas en un microscopio, en nuestro caso las partículas de la substancia, y permiten realizar explicaciones interpretativas y con causalidad. Las simbólicas son las que representan las entidades submicro con letras o signos (Gilbert, 2010).

Para trabajar la visión macro y submicro, y su conexión, nos aproximamos a las ideas de Izquierdo y Aliberas (2004), que consideran que la ACE busca que el estudiante conecte su 
actividad manipulativa y experimental con la teoría y con las formas de hablar. Pensamos que se debe aprender a observar, a interpretar y a predecir, pero también a explicar y discutir lo que se aprende. Por ello, las prácticas de laboratorio se trabajan de manera conjunta e integradas con la teoría; no se consideran como actividades complementarias que pueden realizarse antes o después de las explicaciones teóricas.

Una de las críticas que se hacen a las actividades prácticas es que, incluso cuando los alumnos participan de alguna manera en su preparación o están planteadas totalmente o en parte como investigaciones, no llegan a ver la conexión entre lo que hacen y la teoría, ni el lugar que ocupa el trabajo que se hace en el laboratorio en la validación del conocimiento científico.

Durante la actividad práctica debemos promover que el alumnado establezca puentes entre lo que hace y lo que observa con las ideas científicas (el modelo), porque así podrá interpretar lo que está pasando (Tabla 1). No debemos quedarnos en una situación del tipo "manos en acción", sino que debemos propiciar que el alumnado pase a una situación "mentes en acción" (Millar y Abrahams, 2009).

Tabla 1. Relación entre lo que se hace y observa con la interpretación de lo que se observa (elaboración propia)

\begin{tabular}{|c|c|}
\hline Manos en acción & Mentes en acción \\
\hline ¿Qué hago? & ¿Por qué pasa? \\
\hline Observación & Modelo: interpretación \\
\hline ¿Qué pasa? & Explicarlo en relación al \\
\hline Propiedades & $\begin{array}{l}\text { modelo y a las } \\
\text { propiedades }\end{array}$ \\
\hline
\end{tabular}

\section{Metodología}

\section{Participantes}

Durante el curso 2016/17 se hizo un estudio piloto con 90 alumnos, y en el curso 2017/18 se realizó una investigación con 85 alumnos, todos de 1ำ de la ESO, del Instituto Manuel Blancafort de la Garriga, un municipio de unos 16.000 habitantes de la provincia de Barcelona. En ambos casos, los alumnos estaban repartidos en cuatro grupos-clase de agrupación heterogénea.

En el curso 2016/17 la profesora del grupo A era la primera autora de la investigación. En los otros tres grupos (B, C y D) daba clase una profesora experta, que participó en el diseño de los materiales didácticos. El examen que se realizó este curso no fue preparado para realizar una investigación, sino que era un examen rutinario. Se elaboraron modelos de examen similares, pero con la mayoría de preguntas distintas en relación a los ejemplos propuestos, y se realizaron en días distintos. La decisión de analizar los resultados vino del interés por detectar el nivel de desempeño de los alumnos en la realización de dibujos a nivel submicro y en el uso de las ideas del modelo cinético-corpuscular en las descripciones y explicaciones.

La reflexión sobre los resultados llevó a plantear el estudio que se presenta y a realizar cambios en los materiales, para profundizar en el andamiaje de los aspectos que presentaron mayores dificultades para el alumnado. Además, se revisaron y completaron las ideas del modelo a compartir con los alumnos, que son la base del instrumento de análisis. 
En el curso 2017/18 impartían clase dos profesoras, una novel (en los grupos C y D) y una experimentada (la misma que el curso anterior) en los grupos A y B. Las dos profesoras trabajaron en equipo y en contacto con la investigadora. En este estudio se uniformizó al máximo la recogida de datos: el examen fue el mismo para todo el alumnado y se hizo en el mismo día. Se analizaron las respuestas del examen y, posteriormente, la investigadora discutió los resultados con las dos profesoras participantes. Así se constataron los aspectos que se habían mostrado problemáticos, y se compartieron con el alumnado. Además, surgieron ideas para seguir remodelando las actividades y se facilitó la incorporación de la profesora novel a la metodología empleada, que se siguió aplicando a lo largo del curso.

\section{Actividades}

El tema se inicia con prácticas de dilatación térmica en el laboratorio. Se observa la dilatación térmica de un sólido, una bola metálica que atraviesa o se queda atascada en un aro antes y después de ponerla sobre una placa térmica; de un líquido introducido en un matraz con una pipeta de diámetro reducido en el extremo superior, que sube y baja por la pipeta según su temperatura; y del aire de un matraz y de un globo, encajado en su extremo, que se hincha y deshincha, también alternativamente si está o no sobre la fuente de calor. Previamente, se trabajan los requisitos formales de las definiciones, las descripciones y las explicaciones científicas, a partir de pautas que se proporcionan y se discuten con los alumnos.

Para la realización de definiciones se indica que, en primer lugar, se debe incluir el concepto a definir y después la categoría, unidas por el verbo ser (es/son), y un conector que enlace con las características propias del concepto (Tabla 2). Se comenta con los alumnos y se realiza una definición consensuada, que en este caso fue: la dilatación térmica es un fenómeno físico que consiste en la variación de volumen de una substancia en respuesta a los cambios de temperatura.

Tabla 2. Pauta para realizar definiciones

Concepto + es/son + categoría + conector + características propias del concepto

A continuación, se consensúan las ideas clave de la dilatación térmica (Tabla 3) a partir de las discusiones sobre lo que se observó en el laboratorio (macro) y la interpretación de lo que sucedió a nivel submicro. Se diferencia entre descripciones y explicaciones de fenómenos de dilatación térmica. Las descripciones deben expresar lo que se observa, lo que pasa, pero también se debe indicar la relación entre los cambios de las propiedades observables implicadas en el proceso, en este caso la temperatura y el volumen (Idea 1). En las explicaciones, se debe expresar la relación entre la visión macro y la submicro y utilizar el resto de las ideas del modelo para realizar la interpretación del fenómeno estudiado.

En un trabajo cooperativo en grupos de tres, cada alumno explica la dilatación de uno de los estados, utilizando las ideas clave. También hace un dibujo a nivel submicro, mostrando la separación y movimiento de las partículas antes y después de colocarlo en la fuente de calor. A continuación, los miembros del grupo comparten sus trabajos, hacen correcciones y escriben juntos las semejanzas y diferencias de los tres procesos. En una puesta en común, con todo el grupo-clase y la profesora, se comparte el uso de las ideas clave en los dibujos y explicaciones. Para acabar, el grupo revisa sus producciones, se realizan correcciones y se elabora un póster. 
Tabla 3. Ideas clave sobre dilatación térmica en las substancias (elaboración propia)

Idea 1.-Relación entre los cambios de temperatura y los cambios de volumen de una substancia (macro).

Idea 2.- Separación de partículas en sólidos, líquidos y gases (submicro).

Idea 3.-Movimiento de partículas en sólidos, líquidos y gases (submicro).

Idea 4.-Cambios en las partículas de una substancia (movimiento y separación) como resultado de la transferencia de calor (submicro).

Idea 5.- Relación de la energía cinética de las partículas (submicro) con los cambios en el movimiento y separación de las partículas (submicro).

Idea 6.- Relación de la energía cinética de las partículas (submicro) con el aumento de temperatura y de volumen (macro) y con los cambios en el movimiento y separación de las partículas (submicro).

\section{Datos de la investigación}

Los datos que se analizan corresponden al examen que se hizo al final de la unidad didáctica. En la primera pregunta se pidió a los alumnos que hicieran dibujos submicro para centrar el foco en el modelo cinético-corpuscular. Después se incluyeron preguntas sobre las prácticas de laboratorio, para acabar con preguntas de aplicación (Tabla 4).

Tabla 4. Tipología de las preguntas del examen

\section{Preguntas}

1.-Dibujos a nivel submicro, realizados por los alumnos, sobre la separación y movimiento de las partículas de sólidos, líquidos y gases.

2.-Interpretación de dibujos macro y submicro de la dilatación de un sólido (proporcionados). Descripción y explicación sobre la práctica de la dilatación del sólido: una bola metálica.

3.-Realización de dibujos submicro de la dilatación de un gas. Explicación de la dilatación de un gas a partir de la práctica realizada.

3.-Pregunta sobre la dilatación de un líquido: explicación del funcionamiento de un termómetro de alcohol, no trabajada en clase.

4.-Experiencias en relación a fenómenos de dilatación térmica (aplicación).

\section{Análisis de los resultados}

En los exámenes, se ha estudiado el uso de ideas del modelo en dibujos y respuestas escritas. En los dibujos elaborados por los alumnos, se ha analizado la separación de las partículas y la representación del grado de movimiento. En los dibujos proporcionados, se ha analizado si se reconocía el significado de su simbología y se relacionaba la separación y movimiento de las partículas con los cambios de temperatura y volumen. En las descripciones, se ha diferenciado entre el alumnado que solo indica lo que se observó y el alumnado que relaciona los cambios de temperatura con los de volumen, es decir, los que incluyen la Idea 1. En las explicaciones se ha registrado el número de las ideas clave del modelo utilizadas (Tabla 1) y solo se ha considerado adecuado su uso si la causalidad de las ideas era correcta y su expresión era coherente.

En los ejemplos de respuestas del alumnado que se adjuntan, hemos subrayado los conceptos a nivel macro temperatura y volumen, y escrito en negrita los conceptos relacionados con la visión submicro. 
Pregunta 1.-Dibujos a nivel submicro, realizados por los alumnos, sobre la separación y movimiento de las partículas de sólidos, líquidos y gases.

Se debían realizar dibujos de la separación y movimiento de las partículas de dos sólidos (un cristal de pirita y un vaso de plástico), dos líquidos (agua del grifo y zumo de naranja) y dos gases (helio de un globo y gas de un globo aerostático), de los cuales se adjuntó un dibujo a nivel macro. Este tipo de representaciones se habían hecho en clase con otros objetos de la vida cotidiana y escolar. En la Tabla 5 se adjuntan dibujos de una alumna y en la Tabla 6 se incluyen los resultados del análisis.

Tabla 5. Dibujos que presentan la separación de las partículas de manera diferenciada en sólidos, líquidos y gases, e incluyen una simbología para el movimiento de las partículas en los tres estados

\begin{tabular}{|c|c|c|}
\hline Sólido & Líquido & Gas \\
\hline $\begin{array}{l}0,01 \\
010101 \\
010,01\end{array}$ & $0^{11} 0^{\prime \prime \prime} 0^{\prime \prime} 0^{\prime \prime}$ & $\begin{array}{l}\text { olli oll1 } \\
\text { ollin } \\
\text { ollin }\end{array}$ \\
\hline
\end{tabular}

El 80\% del alumnado (Tabla 6) indicó la separación de las partículas de manera diferenciada en sólidos, líquidos y gases en dibujos similares a los de la Tabla 5. Respecto al movimiento de las partículas, se registraron por separado los resultados de los sólidos, los líquidos y los gases. Un $45,9 \%$ de los alumnos utilizó algún tipo de simbología para representar el movimiento de las partículas en sus dibujos, pero tan solo un $5,9 \%$ lo hizo en los tres estados de la materia.

Tabla 6. Utilización de las ideas clave en dibujos submicro realizados por el alumnado. (En negrita los resultados de las clases de la profesora experimentada)

\begin{tabular}{|c|c|c|c|c|}
\hline Ítems & Resultado & $\begin{array}{c}\text { № alumnos por clase } \\
\text { y totales }\end{array}$ & \multicolumn{2}{|c|}{$\begin{array}{c}\% \text { de los } \\
85 \text { alumnos }\end{array}$} \\
\hline \multirow{2}{*}{$\begin{array}{l}\text { Separación } \\
\text { diferencial de } \\
\text { partículas en SLG }\end{array}$} & No o incongruencias & $8+3+2+4=17$ & \multicolumn{2}{|l|}{$20 \%$} \\
\hline & Sólido, líquido y gas & $17+20+14+17=68$ & \multicolumn{2}{|l|}{$80 \%$} \\
\hline \multirow{4}{*}{$\begin{array}{l}\text { Movimiento } \\
\text { diferencial de } \\
\text { partículas SLG }\end{array}$} & No o incongruencias & $6+5+16+19=46$ & $54,1 \%$ & \\
\hline & Solo gas & $12+9+0+0=21$ & $24,7 \%$ & \multirow{3}{*}{$45,9 \%$} \\
\hline & Líquido y gas & $3+8+0+2=13$ & $15,3 \%$ & \\
\hline & Sólido, líquido y gas & $4+1+0+0=5$ & $5,9 \%$ & \\
\hline
\end{tabular}

Además, los resultados en los grupos de la profesora experimentada (en negrita en la tabla 6) fueron, en general, superiores a los de la profesora novel. Solo dos alumnos de la profesora novel indicaron el movimiento de las partículas (en líquidos y gases) mientras que 37 alumnos de la profesora experimentada representaron este movimiento, al menos en uno de los estados ( 18 en uno de sus grupos y 19 en el otro). Pocos estudiantes mostraron el movimiento de las partículas de los sólidos. Esta diferencia entre grupos según la profesora fue inesperada, ya que el curso anterior no se produjo, y los resultados en este apartado fueron muy superiores. 
Pregunta 2.- Interpretación de dibujos macro y submicro de la dilatación de un sólido (proporcionados). Descripción y explicación sobre la práctica de la dilatación de un sólido: una bola metálica

Los dibujos submicro mostraban la situación y movimiento de las partículas de una substancia; en el de mayor volumen había una mayor separación y movimiento de partículas. Debían indicar el significado de las líneas que había a los lados de las bolas que representaban las partículas y qué dibujo correspondía a una mayor temperatura. El 74\% del alumnado contestó que las líneas representaban el movimiento de las partículas, y relacionó correctamente que el dibujo con mayor movimiento y separación de partículas correspondía a una mayor temperatura y, por tanto, a un mayor volumen de la bola, incluyendo algunas ideas submicro (ver ejemplo).

"El segundo dibujo representa el de mayor temperatura, porque es más grande. Las partículas con el aumento de calor tienen más energía cinética, se mueven más, se separan, chocan entre ellas y se dilata, ocupa más volumen."

A continuación, se les pedía que primero hicieran una descripción de lo que se observaba en los dibujos (visión macro en términos de propiedades) y después que explicaran a nivel submicro por qué pasaba. La mayoría del alumnado $(89,5 \%)$ realizó la descripción y el $69,8 \%$ incluyó la relación entre los cambios de temperatura y de volumen, indicando su correlación (Idea 1), como se muestra en el siguiente ejemplo.

"Pasamos una bola metálica por un aro y luego la sacamos y la pusimos en la placa térmica. Con el aumento de temperatura aumentó el volumen de la bola y luego no pasó por el aro (Idea 1)."

En las explicaciones (Tabla 7), el 89,5\% del alumnado incluyó alguna de las ideas de la dilatación térmica a nivel submicro, aunque sólo el 39,5\% las usó todas, siendo casi el doble el número de alumnos de la profesora experimentada. En ellas, se incluyó la idea de movimiento de las partículas en un porcentaje mucho más elevado que en los dibujos elaborados en la primera pregunta. Aunque el alumnado de la profesora con experiencia superó en un $22 \%$ al alumnado de la novel, muchos alumnos supieron reconocer los cambios de las partículas del sólido en relación a los cambios de temperatura y volumen.

Tabla 7. Utilización de ideas clave en la descripción y explicación de la dilatación de un sólido. Curso 2017/18

\begin{tabular}{|c|c|c|c|}
\hline Ítems & Resultado & \multicolumn{2}{|c|}{$\%$ alumnos } \\
\hline \multirow{3}{*}{$\begin{array}{l}\text { Descripción de los procesos: dilatación } \\
\text { de un sólido (nivel macro) }\end{array}$} & No describen & 10,5 & \\
\hline & Sólo descripción & 19,7 & \multirow{2}{*}{89,5} \\
\hline & Incluyen Idea 1 & 69,8 & \\
\hline \multirow{3}{*}{$\begin{array}{l}\text { Explicación de los procesos: dilatación } \\
\text { de un sólido (nivel submicro) }\end{array}$} & No explican & 10,5 & \\
\hline & Algunas ideas clave & 50 & \multirow{2}{*}{89,5} \\
\hline & Todas las ideas clave & 39,5 & \\
\hline
\end{tabular}

A continuación, se muestran algunos ejemplos, indicando las ideas clave utilizadas en cada caso.

"Esto ha pasado porque las partículas de la bola han recibido calor de la placa y han empezado a moverse más rápido y a separarse y por eso la bola se ha hecho más grande." (Idea 4) 
"Lo que ha pasado es que con el calor de la placa térmica ha aumentado la temperatura y se ha aumentado el volumen, porque las partículas se han movido, han chocado entre ellas y han ocupado más espacio." (Ideas 1, 2, 3 y 4)

"La bola no pasó por el aro después de calentarla, porque las partículas recibieron calor y aumentó la energía cinética y la temperatura. Entonces las partículas se movían más y chocaban entre ellas. Como se separaban ocuparon más espacio y la bola aumentó de volumen." (Todas las ideas)

\section{Pregunta 3.-Dilatación de un gas: realización de dibujos submicro y explicaciones.}

En este caso se adjuntaron dos fotografías que mostraban un matraz con un globo en una placa térmica; en un caso el globo estaba hinchado y en el otro deshinchado. Se pedía que explicaran lo que se observaba, utilizando los conceptos trabajados en el tema, y se incluían algunos ejemplos de conceptos a nivel macro y submicro (partículas, temperatura, energía cinética), con la palabra 'etcétera' al final. Se les pedía también que hicieran dibujos de las partículas de gas en el globo hinchado y deshinchado (Tabla 8).

Se consideraron correctos los dibujos que representaban la separación y movimiento de las partículas en el globo y el matraz, y también los que las mostraban solo en una parte del montaje. Los dibujos con partículas solo dentro del globo también se consideraron correctos, porque así se pedía en la pregunta, ya que no se especificó que debían dibujarse las partículas de gas dentro del globo y del matraz.

El $60 \%$ del alumnado expresó de manera correcta la separación de las partículas del gas (globo hinchado y deshinchado) y el $47 \%$ de los alumnos representó correctamente el movimiento en ambos casos (el $41 \%$, alumnado de la profesora experimentada; el $6 \%$, de la profesora novel). Algunos alumnos hicieron dibujos con todos los requisitos, pero no representaron el movimiento de las partículas en el caso de la temperatura más baja. Otros las dibujaron solo en el matraz, con o sin representación de movimiento en el caso del globo deshinchado.

Tabla 8. Dibujos submicro de la dilatación de un gas realizados por los alumnos

\begin{tabular}{|c|c|c|}
\hline $\begin{array}{c}\text { Sin representación de } \\
\text { movimiento en menor } \\
\text { temperatura }\end{array}$ & $\begin{array}{c}\text { Una parte del montaje, } \\
\text { con separación y } \\
\text { simbología adecuadas }\end{array}$ & $\begin{array}{c}\text { Separación y simbología del } \\
\text { movimiento adecuadas. Mismo } \\
\text { número de partículas. }\end{array}$ \\
\hline
\end{tabular}

Se han considerado correctas las explicaciones que incluyen todas las ideas, y también las que no las expresan todas, pero hacen una interpretación de lo observado coherente y muy completa. El 56\% del alumnado de la profesora experimentada realizó interpretaciones con estas características, pero en los grupos de la profesora novel solo lo hizo el $15 \%$. El problema principal en estos grupos se centró en la energía cinética (Ideas 5 y 6 ), tanto en su inclusión en las explicaciones como en su causalidad. Si no tuviéramos en cuenta esta idea clave, el $55 \%$ de los alumnos de la profesora novel tendría respuestas correctas, número muy similar al de los grupos de la otra profesora. 
En primer lugar, incluimos dos ejemplos de explicación coherente y completa, aunque en el segundo no se explicita una de las propiedades, el volumen.

"Esto ha pasado porque la temperatura ha aumentado, por lo tanto, también ha aumentado la energía cinética y el movimiento de las partículas. Gracias a este movimiento el gas ha aumentado de volumen y el globo ha subido. Sacamos el matraz de la fuente de calor y se deshincha. Esto pasa porque la temperatura ha bajado, la energía cinética ha bajado, y el movimiento de las partículas también. Por lo tanto, el volumen del gas ha disminuido y el globo ha retornado a su estado inicial."

"Hemos enganchado un globo en un Erlenmeyer y hemos aumentado su temperatura con una placa térmica. Al hacerlo el globo se ha hinchado. Ha pasado porque al calentarlo ha aumentado la energía cinética de las partículas, que se han movido más y han chocado entre ellas, separándose y así se han puesto dentro del globo hinchándolo."

A continuación, adjuntamos una explicación que no incluye todas las ideas, indicando las que se utilizaron.

"Pusimos el globo en el matraz Erlenmeyer y estaba deshinchado después lo calentamos con la placa térmica y el globo se hinchó. Esto pasa porque las partículas del gas recibieron energía cinética y se movían más y como las paredes del globo son elásticas, el globo se empezó a hinchar." (Idea 4 e idea 5, pero solo incluye movimiento, no separación)

En muchas explicaciones, como pasa en el ejemplo anterior, en que se obvió la separación de las partículas, sí se representó en los dibujos.

En el siguiente ejemplo vemos una explicación que muestra que se han incorporado aspectos interesantes, como la separación entre lo que se ve y lo que se interpreta, pero la interpretación presenta problemas en la causalidad de la energía cinética (doble subrayado).

"Lo primero que hicimos fue coger un erlemeyer y ponerle un globo encima. Después lo pusimos en la placa térmica y al cabo de un rato el globo se hinchó. ¿Por qué? Esto pasó porque las partículas de dentro del globo (gas), como todas las otras, al notar calor, comenzaron a vibrar y a chocar, produciendo así la energía cinética. Cuando esto pasó, no tenían otro sitio al que ir que al globo, haciendo que este se hinchara.

Pregunta 4.-Pregunta de aplicación sobre la dilatación de un líquido: explicación del funcionamiento de un termómetro de alcohol, no trabajada en clase

La dilatación de los líquidos se incluyó en una pregunta de aplicación. En un dibujo adjunto se mostraba un niño en la cama con un termómetro en la boca. Se indicaba que la doctora que lo había ido a visitar le había puesto un termómetro de alcohol para saber si tenía fiebre. Se pedía que se explicara cómo marca el termómetro la temperatura, teniendo en cuenta la dilatación de los líquidos. Se decidió hacerlo de esta manera porque, más adelante, el uso del termómetro y la comprensión de su funcionamiento serían claves en la comprensión de los fenómenos estudiados en las prácticas de laboratorio sobre las adaptaciones de los animales a la temperatura.

En este caso, el $70,6 \%$ del alumnado utilizó algunas $(40,6 \%)$ o todas las ideas clave (30\%) en sus razonamientos, y el porcentaje de alumnado de la profesora experimentada que realizó explicaciones bien fundamentadas a partir del modelo fue sólo ligeramente superior al de la profesora novel. 
Algunas respuestas se centraron solo en la situación que se planteaba (primer ejemplo), pero en el $15,3 \%$ de las explicaciones se relacionó con la práctica de la dilatación del líquido (en negrita y subrayado en el segundo ejemplo), que actuó de andamiaje para la explicación.

"El termómetro se calienta gracias a nuestro calor, que hace que las partículas adquieran energía cinética y se dilate el alcohol. Si tenemos fiebre estamos más calientes por eso el alcohol marca más temperatura, ya que nosotros damos a las partículas más energía cinética."

"El termómetro marca la temperatura porque las partículas absorben la energía cinética hasta un punto en que ya no pueden más porque están a la temperatura del cuerpo del niño. Al calentarse el alcohol sube igual que en la pipeta, midiendo así la temperatura."

Finalmente, un ejemplo donde se expresa la idea de equilibrio térmico, no trabajada en clase.

"El alcohol se calienta y las partículas (del alcohol) van subiendo por el termómetro, pero sólo hasta un cierto punto. Suben hasta estar a la misma temperatura que el niño, porque las partículas ya no reciben más energía cinética, porque él les da hasta un cierto punto de calor".

Pregunta 5.-Experiencias y opiniones del alumnado en relación a fenómenos de dilatación térmica (aplicación)

En la última pregunta se pidió a los alumnos que relataran, desde el punto de vista científico, alguna situación que les hubiera pasado en su vida cotidiana relacionada con la dilatación térmica. Solo la contestaron el $20 \%$ de los alumnos. Como dato a destacar, el $26 \%$ del alumnado de la profesora novel explicó de manera adecuada, y en ocasiones extensa, algún fenómeno de su vida personal; en cambio, solo un $6 \%$ del alumnado de la profesora experimentada lo hizo.

También se observó que las respuestas de los estudiantes de la profesora experimentada eran más extensas. Quizás los alumnos de la profesora novel tuvieron más tiempo para pensar en fenómenos que les habían ocurrido, al dedicar menos tiempo a sus respuestas anteriores.

"Como nosotros tenemos partículas, en verano con el calor a veces nos hinchamos y a mí me pasó que primero el reloj me iba un poco ancho y después me apretó un poco."

"Cuando pones una pizza (o un pastel) al horno recibe calor y las partículas se separan (se mueven, vibran) y aumenta de volumen."

"Un día de verano estábamos haciendo una barbacoa y dejamos las llaves al sol y cerca del fuego y se dilataron. Las cogimos con guantes y no cabían en el agujero de la cerradura y nos quedamos encerrados en la terraza hasta que se enfriaron."

\section{Conclusiones}

Los resultados muestran diferencias en relación al nivel de desempeño en la utilización del modelo cinético-corpuscular en dibujos, descripciones y explicaciones por parte del alumnado. También revelan diferencias inesperadas entre los resultados de la profesora novel y la experimentada, tanto en la realización de dibujos como en las explicaciones, ya que en el estudio piloto no se produjeron diferencias destacadas entre grupos-clase. 


\section{Dibujos: realización e interpretación}

Las demandas cognitivas de un dibujo y un texto escrito no son las mismas, ya que se trata de dos modos comunicativos distintos. En el escrito, los alumnos pueden decir que las partículas se mueven más o menos, pero en el dibujo es necesaria una simbología que lo muestre.

Representar la separación de las partículas en dibujos a nivel submicro no presentó dificultades para la mayoría de los alumnos, pero sí las hubo en la representación de su movimiento, especialmente en el alumnado de la profesora novel. Revisando los resultados, se constató que la profesora novel no incluyó una simbología para expresar el movimiento de las partículas en los dibujos que hizo en clase, hecho que pone en evidencia la influencia de la instrucción en los resultados de los alumnos.

Por otro lado, pocos alumnos representaron la vibración de las partículas de los sólidos. Datos que contrastan con que la mayoría supo interpretar el movimiento de las partículas de una bola metálica sobre un dibujo proporcionado y muchos lo incluyeron en sus explicaciones. El dibujo proporcionado, en este caso, ayudó a elaborar explicaciones y a interiorizar maneras de simbolizar fenómenos.

Coincidimos con Uchinokura (2018) cuando afirma que plasmar lo que se observa en un dibujo submicro es un proceso complejo, y que la transición entre la visión macroscópica y submicroscópica es difícil. En este sentido los resultados muestran que la autoevaluación y coevaluación de los dibujos pueden mejorar las producciones de los alumnos. Así mismo, introducir dibujos ya elaborados en los ejercicios de clase con el objetivo de conocer el lenguaje propio del modo visual, puede facilitar la adquisición de recursos para plasmar en un dibujo personal el comportamiento de las partículas. En el examen, los resultados de la pregunta de interpretación de dibujos proporcionados de la dilatación térmica fueron satisfactorios, así como los de la descripción e interpretación del proceso que se pedían a continuación.

En el caso de elaboración de dibujos personales, que debían relacionar la visión macro con la submicro en fenómenos de dilatación de gases, se detectaron problemas en la representación del movimiento de las partículas, pero no en la separación, y en la idea de movimiento de partículas en el gas a menor temperatura. Lo que indica una mayor facilidad para representar la estructura de los gases que su dinámica.

Una implicación de estos resultados es revisar de nuevo las actividades para poner el foco en la idea de que las partículas siempre están en movimiento, también en los sólidos y también a temperaturas bajas.

\section{Descripciones y explicaciones}

Consideramos que las actividades prácticas realizadas en el laboratorio han ayudado a los alumnos a iniciarse en la diferenciación de lo que se observa a nivel macro (descripciones) y la interpretación de lo que está pasando a nivel submicro (explicaciones).

En muchas descripciones, los alumnos incluyeron los cambios de propiedades macro y su relación, hecho que demuestra la eficacia de la revisión iterativa de los materiales didácticos, ya que al detectarse esta dificultad el curso anterior se modificaron las actividades para trabajar este aspecto (Hernández y Pinto, 2009).

Respecto a las explicaciones, gran parte del alumnado usó, en mayor o menor medida, ideas clave del modelo (visión submicro) para interpretar los fenómenos y las relacionó con lo observado en el laboratorio, y en menor medida, con su vida cotidiana. Además, 
se detectó que en las explicaciones del alumnado de la profesora novel había problemas concretamente con la causalidad de la energía cinética en las Ideas 5 y 6 .

A la vista de los distintos resultados según las profesoras, se revisaron conjuntamente los exámenes y se constató que la profesora novel tenía problemas con la causalidad en relación a la energía cinética y, en consecuencia, los tenían sus alumnos. Hecho que muestra que el análisis de las producciones de los alumnos debe ir acompañado de la revisión de los materiales utilizados y de su implementación.

\section{Fortalezas y debilidades}

El presente estudio está circunscrito a unos alumnos, unas profesoras y un instituto determinados, con un contexto específico, distinto al de otros lugares. Por ello, los resultados no son extrapolables, pero pensamos que la metodología empleada puede ser una buena aproximación para que los alumnos se inicien en la modelización de fenómenos de dilatación térmica y en el uso del modelo cinético-corpuscular en el marco de las prácticas científicas.

El hecho de analizar los resultados en equipo (profesoras e investigadora) ayudó a detectar puntos fuertes y debilidades en la elaboración e implementación de los materiales, y a decidir dónde debía incluirse más andamiaje para facilitar la incorporación de los alumnos al modelo. También permitió revisar con el alumnado aquellos aspectos que se habían mostrado más complejos.

Los resultados muestran que los alumnos se han iniciado en la modelización de fenómenos de dilatación térmica, pero que es necesario ir revisando y completando las ideas clave del modelo cinético-corpuscular a medida que se adquieran nuevos conocimientos, ya que llevan asociadas muchas concepciones alternativas, difíciles de superar (Domínguez Castiñeiras et al, 1996).

La realización de dibujos a nivel submicro es muy importante dentro del proceso de modelización, pero comporta diversos retos. Coincidimos con Uchinokura (2018) en que reflexionar sobre el mismo fenómeno desde distintos niveles de representación puede ayudar a realizar la transición de la visión macro a la submicro, tanto en los dibujos como en las explicaciones.

\section{Reflexiones finales}

Pensamos, tal como propone Osborne (2014), que el objetivo de la enseñanza de las ciencias es desarrollar una manera de entender las ideas existentes sobre el mundo natural ayudando al alumnado a comprender el cuerpo de conocimiento antiguo, consensuado y bien establecido. Si involucramos a los alumnos en el desarrollo de las prácticas científicas, les estaremos dando una nueva manera de mirar, que les proporcionará unas bases científicas para enfrentarse al mundo que les rodea. Conocer las múltiples maneras que se utilizan en los estudios científicos para explorar e intentar entender cómo funciona el mundo natural (Krajcik y Merrit, 2012) les permitirá, a la larga, interpretar fenómenos y tomar decisiones fundamentadas: tomar o no un medicamento, qué coche comprar, poner una vacuna o no, qué tipo de alimentación seguir, opinar sobre el cambio climático o la homosexualidad y un largo etcétera.

Estamos de acuerdo con John Postgate (1995) que "la compresión científica cambia vidas" y que "es (o puede ser) una experiencia cultural tan valiosa, formativa y amena como absorber una gran obra artística o literaria" (p. 8). Pensamos que el doble reto es que personas a priori no interesadas por la ciencia adquieran esta aproximación, este gusto por 
la ciencia, y que las personas que ya tienen una inclinación científica puedan desarrollarla y completarla, de manera que no se pierdan por el camino. Consideramos que puede ser una buena aproximación proporcionar actividades que permitan trabajar las prácticas científicas y faciliten que el alumnado, poco a poco, vaya adquiriendo una manera de trabajar y una manera de mirar el mundo que vaya forjando su cultura científica.

\section{Agradecimientos}

A Mã Elena Gayán Rico y Roser Pratdesaba Moreno, las profesoras participantes, y al Instituto "Manuel Blancafort" de la Garriga (Barcelona). Al grupo LIEC de la UAB.

Investigación financiada por el Ministerio de Economía y Competitividad (con referencia EDU2015-66643-C2-1-P) y realizada en el marco del grupo consolidado ACELEC reconocido por la AGAUR (con referencia 2017SGR1399).

\section{Referencias bibliográficas}

Achieve (2013). Next generation science standards (NGSS): For states, by states. Washington, DC: National Academies Press.

Adúriz-Bravo, A. y Izquierdo-Aymerch, M. (2009). Un modelo de modelo científico para la enseñanza de las ciencias naturales. Revista Electrónica de Investigación en Educación en Ciencias, 4 (3), 40-49.

Benarroch, A. (2000). Del modelo cinético-corpuscular a los modelos atómicos. Reflexiones didácticas. Alambique, 23, 95-108.

Domínguez Castiñeiras, JM., García-Rodeja Fernández, E., Illobre González, ML., Castro Fucci, M., García, S. y Rocha, A. (1996). La naturaleza corpuscular de la materia y su utilización en el campo conceptual calor y temperatura. Caderno Brasileiro de Ensino da Física, 13(1), 11-31.

Garrido Espeja, A. (2016). Modelització i models en la formació inicial de mestres de Primària des de la perspectiva de la pràctica científica. Tesis doctoral. Universidad Autónoma de Barcelona.

Gilbert, JK. (2010). The role of visual representations in the learning and teaching of science: An introduction. Asian-Pacific Forum on Sicence Learning and Teaching, 11(1). Recuperado de: https://www.eduhk.hk/apfslt/download/v11_issue1_files/ foreword.pdf.

Hernández, M. I. y Pintó, R. (2009). Desarrollo iterativo de una secuencia de enseñanza y aprendizaje sobre propiedades acústicas de los materiales. Enseñanza de las Ciencias, no extra, 3564-3568.

Izquierdo, M., Sanmartí N. y Espinet, M. (1999). Fundamentación y diseño de las prácticas escolares de ciencias experimentales. Enseñanza de las Ciencias, 17(1), 45- 59.

Izquierdo, M. y Aliberas, J. (2004). Pensar, actuar i parlar a classe de Ciències. Per un ensenyament de les ciències racional i raonable. UAB. Servei de Publicacions. Departament de Didàctica de la Matemàtica i de les Ciències Experimentals.

Johnson, Ph., y Papageorgiou, G. (2010). Rethinking the Introduction of Particle Theory: A Substance-Based Framework. Journal of Research in Science Teaching, 47(2), 130-150. 
Krajcik, J. y Merritt, J. (2012). Engaging students in scientific practices: What does constructing and revising models look like in science classrooms? Science and Children, 49(7), 10-13.

Lemke, J. (1997). Aprender a hablar ciencia. Lenguaje, aprendizaje y valores. Barcelona: Paidós.

Millar, R. y Abrahams, I. (2009). Practical work: Making it more effective. School Science Review, 91(334), 45-50.

Nebot, MR. y Márquez, C. (2017). Uso del modelo cinético-corpuscular en explicaciones sobre fenómenos naturales. Enseñanza de las Ciencias, no extra, 4287-4292.

Osborne, J. (2014). Teaching Scientific Practices: Meeting the Challenge of Change. Journal of Science Teacher Education, 25, 177-196.

Postgate, J. (1995). Las fronteras de la vida. Barcelona: Ed. Grijalbo Mondadori.

Uchinokura, S. (2018). Students' use of representation and particle models for dissolution. ESERA 2017 Conference, 400-411. 
